\section{Updates on palliative care: what, for whom, and how?}

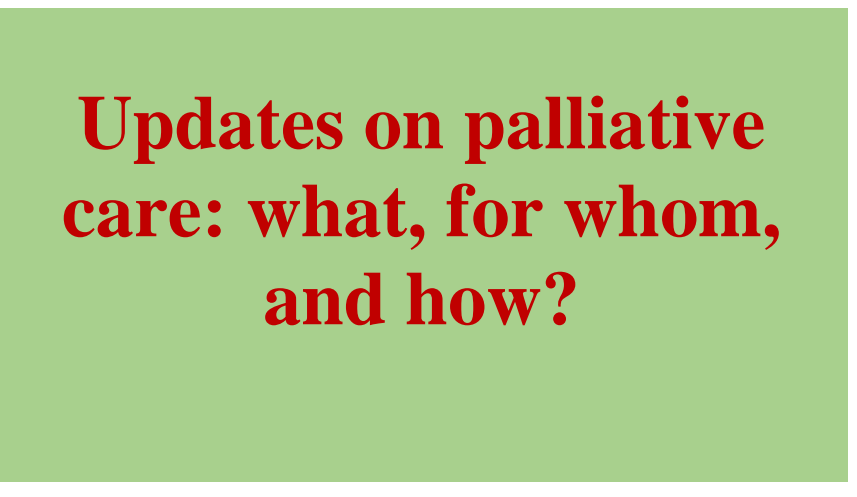

\section{Dr. Khamis Elessi ${ }^{1 *}$}

\author{
${ }^{1}$ Neurorehabiliation \& Pain Medicine \\ Consultant \\ * Corresponding author \\ E-mail address: khamis_essi@yahoo.com
}

https://doi.org/10.33976/IUGNS.29.1/2021/1

\begin{abstract}
:
Palliative care is a new specialty that focuses on relieving pain and other symptoms in patients with serious chronic diseases regardless the diagnosis or the stage of the disease. Palliative care professionals aspire to improve the quality of life for the affected patients, their families and caregivers. This type of care is provided alongside with curative treatment and other interventions that the patient may receive. This study came as a review of the current literature published in scientific peer-reviewed journals and presented as a summary of these studies. This article aims to provide an update on the latest definitions and concept of palliative care, as well as to highlight the various aspects and dimensions of palliative care and the nature of patients who need palliative care.

In summary, we can say that palliative care adds a new dimension to health care that is concerned with the quality of life of the patient and his family. Palliative care is not only restricted to cancer survivors but also cares for all patients with chronic diseases that have no cure but their agonizing symptoms can be managed or at least reduced.
\end{abstract}

Keywords: Palliative care - cancer survivors .

\title{
Background
}

Palliative care is a relatively new field of modern medical care that focuses on relieving and preventing the suffering for patients with cancer and other serious and chronic diseases in as such is an ancient art given a new framework and backed by a body of evidence and experience. Palliative care was defined in 1990 by the WHO followed by specific guidelines for children in 1998 and a revision in 2002 where the scope of palliative care was broadened and included a public health approach (Sepúlveda, Marlin et al. 2002). In 2014 the $67^{\text {th }}$ World Health Assembly approved an important Resolution to ask all signatory countries to ensure palliative care is 
provided and relevant training and planning is integrated. The scope and place of palliative care continues to be developed. A significant step was the publication of the Lancet Commission on Pain and Palliative Care (Knaul F et al, 2018) which sought to define a new term, Serious Healthrelated Suffering, and then to quantify total serious health-related suffering and conceive of a potential new measurement tool; serious health related suffering adjusted life years (SALYS). These publications explore the impact of palliative care that is not limited to one or 2 diagnoses such as cancer or HIV/AIDS but rather relevant when a person and their caregivers are living with chronic illness or another illness that causes serious health related suffering. The response to this suffering needs to be holistic and include many health and social care workers as well as community mobilization. This framework to quantity pain and palliative care needs reveals staggering levels of unmet need and shines a light on one of the biggest health inequalities of our time. A new consensus definition requested by the WHO was published in 2020 (Radbruch L et al) following a rigorous 3 step consensus process gives us the most complete description and highlights the holistic nature of palliative care, the emphasis on quality of life and the focus of care being the patients and the family and caregivers. The majority of adults who need palliative care have chronic cardiovascular disease $(38.5 \%)$, cancer $(34 \%)$, chronic respiratory disease (10.3\%), AIDS (5.7\%) and 4.6\% of them have diabetes (WHO, 2018). Others have kidney failure, chronic liver disease, and degenerative neurological diseases like multiple sclerosis, and dementia. Children with congenital malformations, cerebral palsy and muscular dystrophies or multiple disabilities due to brain or spinal cord injury also need palliative care. Although the concept of palliative care is not new, most doctors have previously focused on treating patients using traditional methods only and some even viewed treatments used to relieve severe pain and other symptoms as dangerous because they had concerns about causing addiction and other unwanted side effects. Palliative care has also been seen as only for end of life care and the holistic aspects not emphasized.

\section{Maintaining Quality despite progressive global aging}

However, during the last twenty years, the focus on patient's quality of life rather than ordinary medical care has increased dramatically. This new focus is gaining more importance in light of the global aging of the baby boomer generation which is contributing to a growing population of patients who are living longer with serious and chronic illness. Taking the concept of serious health related suffering and projecting likely numbers we can see a huge increase in those needing 
palliative care. Increasing age, demographic transitions and the rise of multi-morbidity will all contribute. (Sleeman et al) Figure 1 and 2 below show the projected evolution of burden of serious health-related suffering in World Bank income regions until 2060.

Looking at the USA, the number aged 65 and older is projected to nearly double from 52 million in 2018 to 95 million by 2060, and the 65-and-older age group's share of the total population will rise from 16 percent to 23 percent by 2030 .

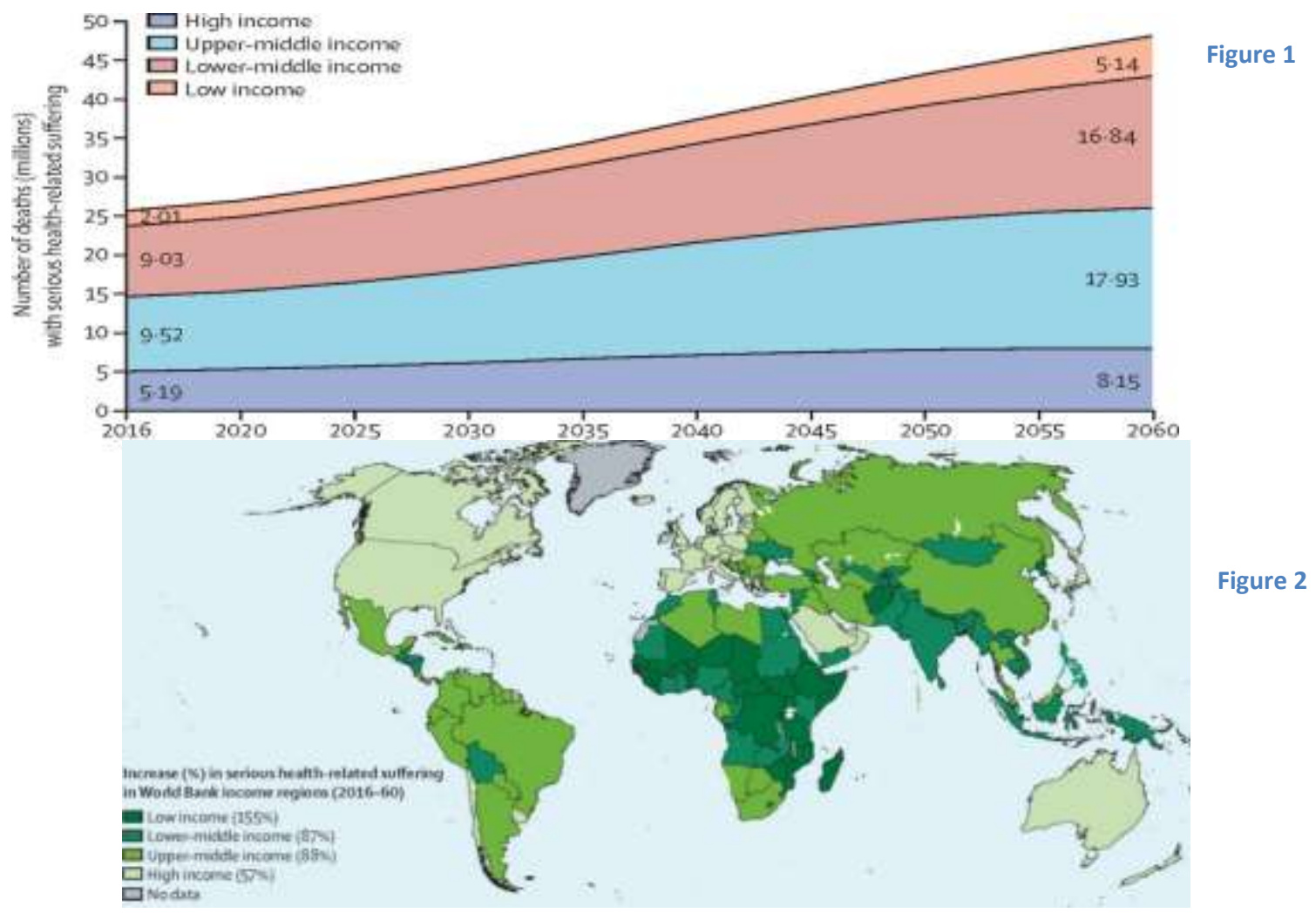

The baby boomer generation (The Elderlies) will make a historical change in USA population in which the older people will outnumber children (U.S. Census Bureau, 2018). This older population will live with chronic disease for longer (Center to advance Palliative Care, 2015) and the pattern of multi-morbidity is changing. More than 5 million Americans now living with Alzheimer's and by 2050, this number is expected to rise to around 14 million (Alzheimer's Association, 2019). This increase of sick elderlies will pose new threat to the world's economy and governments' institutions including hospitals (Mather, Scommegna and Kilduff, 2019). 


\section{Palliative Care \& End of life}

Palliative care should be integrated throughout the illness journey alongside other therapeutic interventions even from the time of the initial diagnosis of advanced and life-threatening diseases. Consequently, the old notion that palliative care begins only after all life-prolonging treatment care has been exhausted and is no longer valid. (Meghani 2004).

Advance care planning is crucial to enable people to have the information they wish to make decisions about their care including treatment interventions and end of life care.

A Canadian study by Daren and colleagues, 2006 explored what seriously- ill and hospitalized patients and their family members consider as the key elements of quality end-of-life care. They focus on the priorities for those patients and their family members who had advanced disease and a prognosis of less than 6 months. The study revealed that crucial discussions were often missing with the doctor. The top priorities were trust and confidence in the attending physician; avoiding recourse to life-support when there is little hope of a meaningful recovery; honest information and communication; being able to address unresolved issues, prepare for life's end and say goodbye. There were significant differences between family members and the patient as well as across the disease spectrum emphasizing the need for open, effective communication and advanced care planning.

Recent studies exploring the impact of early palliative care with oncology patients (Temel JS et al 2010 and 2017 and Zimmerman C et al 2014) improved quality of life, reduction in depressive symptoms, enhancing coping with prognosis and communication about end of life care preferences, greater satisfaction and although less use of interventional oncology there was not shortening of life span.

\section{Integrated Palliative Care Services}

Palliative care uses multiple and integrated methods to care for patients, by relying on the inputs obtained from doctors, pharmacists, nurses, social workers, psychologists, clerics and other health professionals who collectively develop a care plan to alleviate suffering in all areas of the patient's life (see figure 3 below). This multidisciplinary method allows the palliative care team to deal with the physical, emotional, spiritual and social matters that appear as the disease progresses and to support the family in their bereavement.

Figure 3 Integrated model of palliative care

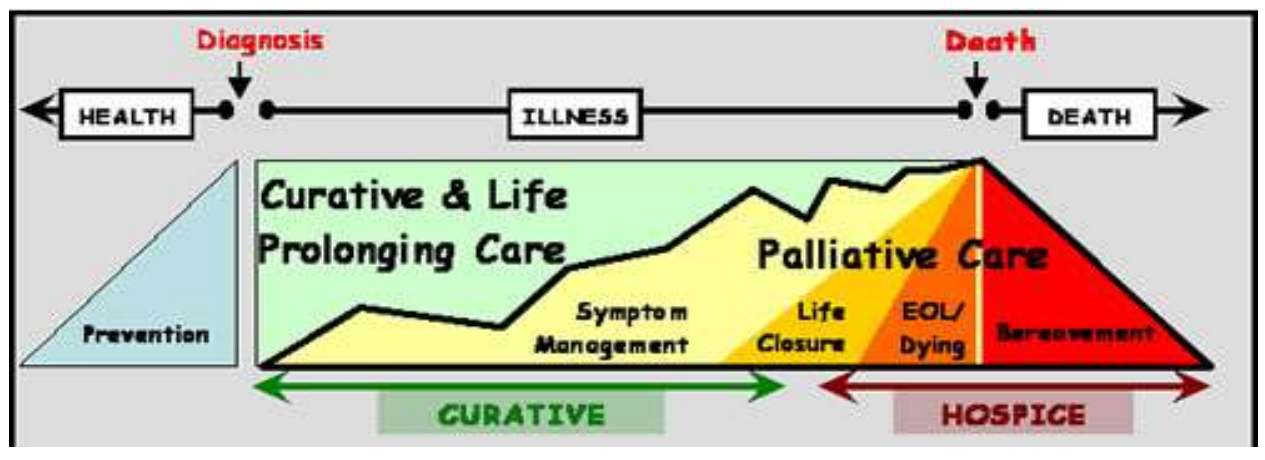


The family still needs support when they lose the patient, whether it is a child or an adult (Mounseyet al, 2018). This multidisciplinary approach can be offered in all care settings and will mostly be the domain of primary care or integrated within specialty settings such as care of the elderly, oncology or internal medicine. Specialist palliative care providers should be available to support complex management and to give advice and training.

\section{Aspects of Comprehensive Palliative Care}

\section{First: physical Care}

\section{Pain Management}

Comprehensive pain management includes treating the reversible using pharmacological and nonpharmacological treatments. Assessment can include appropriate tools such as the 10 point visual analogue scale while remembering that pain is both a sensory and emotional experience. For nonverbal adults or children specific tools can be used like the behavioral observation and adjusted visual analogue scales such as the Wong Baker faces scale. Reassessment is always essential

Pain is the commonest symptom reported in most advanced illnesses (eg. cancer, musculo-skeletal disorders, liver, kidney, pancreas or lung disease complications, etc...), however, pain is often poorly assessed and managed (Natoli, Lazzari and Dauri, 2015)

The experience of pain in a cancer patient is due to organic causes like compression on other organs by cancer or could be due to inorganic causes such as psychological (anxiety, sadness and depression leading to psychological pain), social causes (concern about family, losing his job, inability to play his role at home and abroad with the social stigma) and spiritual factors (feeling guilty, looking at disease as a punishment from God, feelings of hopelessness and abandonment and loss of connection). All of these factors can intertwine with patients' condition and result in pain. But the organic causes remain the main factor causing pain. (Snaman: 2018). Pain could also arise follow therapeutic interventions such as Chemotherapy or radiotherapy.

The palliative care team works to identify the sources of pain, and then alleviate it through pharmacological and non-pharmacological approaches. A wide range of medications are used with a stepwise approach which start with simple analgesics such as acetaminophen (paracetamol) and (aspirin) and other anti-inflammatory drugs, to opioids for moderate pain such as codeine and 
tramadol to opioids for severe pain such as morphine, fentanyl, methadone and hydromorphone. Adjuvant medications like steroids, antidepressants, antiepileptic, and muscle relaxants, can help. With this array of medications a patients' treatment must be individualized, weighing up the risks and benefits of medication, while considering patient comorbidities (Wood et al. 2018). There are other approaches such as surgical or radiological intervention and the whole range of rehabilitation support which are crucial in chronic pain management.

\section{Symptom management (other than pain)}

The patient may experience a number of symptoms caused by the disease and varying in severity. These symptoms include loss of appetite, shortness of breath, nausea, vomiting, constipation, general weakness, bladder problems, mental disorder, etc.

In most cases these symptoms can be controlled in whole or in part via a teamwork which contribute to providing comfort to the patient and his family (see Box 1 below).

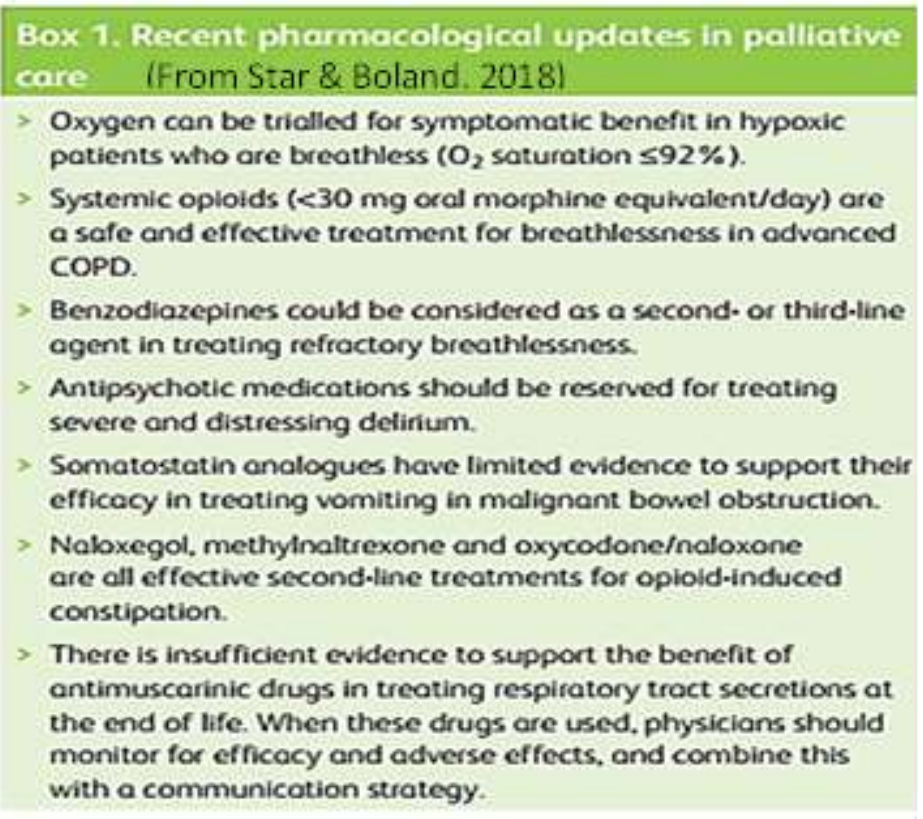

As in pain management, other symptoms should be approached using a combination of careful assessment of the underlying causes, physiology, appropriate investigations, and adopting a holistic management that includes effective communication alongside pharmacological and nonpharmacological interventions. The scope of this paper does not allow detailed discussion of the management of each symptom but the evidence base is expanding. One example is a recent Randomized Clinical Trial to assess the effectiveness of Olanzapine for nausea and vomiting 
among patients with advanced cancer have demonstrated effectiveness for the prevention and treatment of chemotherapy-induced nausea and emesis as well as treatment of chronic nausea and vomiting unrelated to treatment. (Navari, R., 2020

\section{Second: psychosocial care}

Distress is a common feature of chronic illness and it is important to effectively assess the symptoms and impact of distress, recognize and validate normal responses and flag up where further management may be needed. Anxiety, sadness, depressive symptoms and exacerbation of other mental health problems are all common. Building resilience and supporting patients and families is essential through the different stage of chronic illness including diagnosis, treatment, relapse and end of life care. It is important to recognize the profound social impact of chronic illness and its contribution to distress. Again, careful assessment and appropriate involvement of community supports and social work interventions is core to holistic care. For children either as patients or family members play and education are crucial components of care and should not be neglected. Play may also be the medium where children can express their needs as well as a way to build connection and work through distress. Using an empowerment model many of these interventions can be offered by families, trained volunteers or the treating team but further referral for psychological or psychiatric help including medication may be needed. (Morrison and Meier2014)

\section{Third: spiritual Care}

Perhaps the area where many health care workers feel uncomfortable is the spiritual domain. In order to explore we need to have a framework for understanding spiritual distress and spiritual strength and ways to open up this area for assessment. A search for meaning and purpose, to forgive and be forgiven, to be connected in relationships and identity and relate to a higher presence or to God within a faith context are all important components. A question like 'where do you find hope or strength in difficult times?' may allow the person to tell you what is important to them and help identify there sources of spiritual pain. Faith based supports may be crucial for many and links can be made or even a pastoral care person within the palliative care team. 


\section{Communication skills:}

Perhaps the most crucial skill to deliver effective palliative care is communication. Understanding the verbal and non-verbal skills needed, being proficient in active listening, showing empathy, being able to structure and support assessment and offered information and to support decision making are crucial components. The patient may well be the focus of initial communication but it should also include the wider family as appropriate. This is particularly relevant in children where facilitating family and child-focused communication is essential in coordinating care.

Communication should take into account the age and developmental stage of the child as well as recognize the importance of addressing a child's needs directly as well as addressing the needs of caregivers and family.

\section{Implementing palliative care}

As we have discussed there is a significant gap between need and delivery. A summary of the key issues for implementation are included in the recent consensus definition (Radbruch et al 2020) (Table 1).

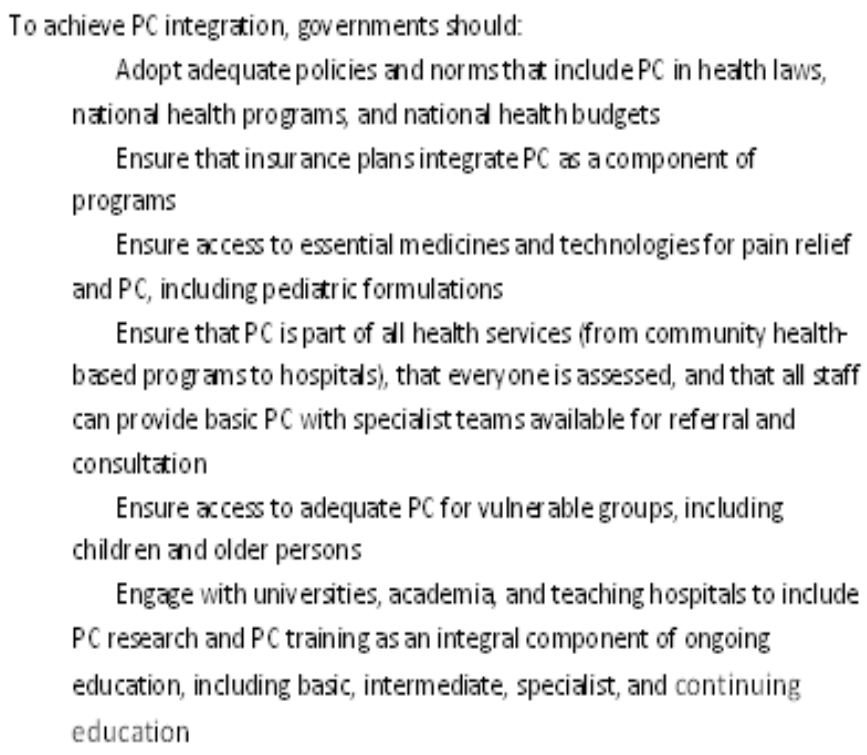

Table 1
This emphasizes the need for comprehensive competency based education and training as well as policy and planning throughout the health system with a particular focus on vulnerable populations. The latter includes growing work examining the interface between humanitarian settings and refugees and palliative care. (WHO 2018).

\section{Palliative care in the Middle East}

Unfortunately in most of the Middle East and in third world countries, palliative care has not been fully integrated into all health care systems, despite evidence that it increases quality of life 
outcomes and reduces end-of-life suffering. In the Middle East, it is provided by various institutions, ranging from voluntary groups and NGOs to formal hospitals and hospital units .One of the biggest obstacles to establishing palliative care programs is the lack of training and awareness of palliative care among health professionals, an impediment to improving access to this service. There is another obstacle as palliative care professionals struggle to secure financing, and to establish basic education for palliative care, in order to raise awareness of this specialization.

Despite this bleak picture, there are some achievements, as some individuals and institutions in this field have achieved notable successes since the early 1990s, after the opening of the first palliative treatment unit in the Kingdom of Saudi Arabia in 1992, and Jordan and Lebanon now have official and specialized programs for doctors, while Egypt, Jordan, Oman, Qatar, and Palestine have developed other advanced training (such as specialized masters or diplomas or training courses in palliative care). In Lebanon, it was announced that at least one palliative care unit was established to teach medical colleges as a separate topic, and Egypt and Kuwait decided to teach palliative care. However, some countries, including Yemen, Libya and Syria, have little or no palliative care activity due to political issues that can affect the availability of resource alongside with a lack of education and awareness. (Ghaly et al, 2018)

Palliative care is one of the most interesting and growing areas of health care encompassing holistic care, a growing evidence base, broad range of diseases and ages, pharmacological and non-pharmacological interventions, teamwork, a focus on vulnerable populations and the opportunity for a deepening therapeutic relationship from the health care provider based on compassion and shared humanity.

\section{References}

- Alzheimer's Association (2019) Alzheimer's Disease: Facts and Figures. Alzheimers Dement 2019;15(3):321-87.

- Balboni TA, Fitchett G, Handzo GF, Johnson KS, Koenig HG, Pargament KI, et al.(2017) State of the science of spirituality and palliative care research part II: Screening, assessment, and interventions. J Pain Symptom Manage, 54:441-53

- Center to advance Palliative Care (2015) America's Care of Serious Illness: 2015 State-ByState Report Card on access to Palliative care in our nation's hospitals. www.npcrc.org 
- Daren K. Heyland, Peter Dodek, \& Graeme Rocker et al. (2006) What matters most in endof-life care: perceptions of seriously ill patients and their family members, CMAJ 174 (5) 627-633; DOI: https://doi.org/10.1503/cmaj.050626

- Etkind SN, Bone AE, Gomes B, et al. (2017) How many people will need palliative care in 2040? Past trends, future projections and implications for services. BMC Med.J. 15: 102

- Ghaly M, Diamond RR, El-Akoum M, Hassan A. (2018) Palliative care and Islamic ethics: Exploring key issues and best practice. Doha, Qatar: World Innovation Summit for Health, ISBN: 978-1-912865-04-8.

- Joanne Lynn (2004). Sick to death and not going to take it anymore: reforming health care for the last years of life. Berkeley: University of California Press. Page 72. ISBN 0-520-24300-5

- Knaul F, Bhadelia A, Rodriguez N, Arreola-Ornelas H, Zimmermann C. (2018) The lancet commission on palliative care and pain relief - Findings, recommendations, and future directions. Lancet Global Health;6:S5-6.

- Mather Mark, Scommegna Paola , Kilduff Lillian (2019) "Aging in the United States,". Population Reference Bureau's Population Bulletin July 15, 2019.

- Meghani, S. H. (2004). "A concept analysis of palliative care in the United States." Journal of advanced nursing 46(2): 152-161.

- Morrison, R. S. and D. E. Meier (2004). "Palliative care." New England Journal of Medicine 350(25): 2582-2590.

- Mounsey, L., Ferres, M., \& Eastman, P. (2018). Palliative care for the patient without cancer. Australian journal of general practice, 47(11), 765-769.

- National Cancer Institute (NCI) Staff (2016). "Study Confirms Benefits of Early Palliative Care for Advanced Cancer". Available at https://www.cancer.gov/news-events/cancercurrents-blog/2016/palliative-care-quality. Accessed on april 20, 2020.

- Natoli S, Lazzari M, Dauri M, (2015) Open questions in the treatment of cancer pain: time for a strong evidence-based approach? Expert Opin Pharmacother. 2015 Jan; 16(1):1-4.

- Navari, R. (2020) Nausea and Vomiting in Advanced Cancer. Curr. Treat. Options in Oncol. 21, 14. doi.org/10.1007/s11864-020-0704-8

- Puchalski C, Ferrell B, Virani R, Otis-Green S, Baird P, Bull J, et al. Improving the quality of spiritual care as a dimension of palliative care: The report of the consensus conference. $\mathrm{J}$ Palliat Med 2009;2:885-904.

- Radbruch L, Knaul F, de Lima L, de Joncheere C, Bhadelia A. (2020) The key role of palliative care in response to COVID-19 tsunami of suffering. Lancet 395:1467-9.

- Radbruch L, De Lima L, Knaul F, Wenk R, Ali Z, Bhatnaghar S, et al. (2020) Redefining palliative care - A new consensus-based definition. J Pain Symptom Manage.J. pii: S08853924(20)30247-5

- Sepúlveda, C., et al. (2002). "Palliative care: the World Health Organization's global perspective." Journal of pain and symptom management 24(2): 91-96.

- Seymour, J. E (2004). "Morphine use in cancer pain: from 'last resort' to 'gold standard'. Poster presentation at the Third research Forum of the European Association of Palliative Care". Palliative Medicine. 18 (4): 378. 
- Sleeman KE, Brito M, Etkind S, Nkhoma K, Guo P, Higginson IJ, Gomes B, Harding R (2019) The escalating global burden of serious health-related suffering: projections to 2060 by world regions, age groups, and health conditions

The Lancet Global Health July

- Snaman, J. M., Kaye, E. C., Baker, J. N., \& Wolfe, J. (2018). Pediatric palliative oncology: the state of the science and art of caring for children with cancer. Current opinion in pediatrics, 30(1), 40-48.

- Star A. and Boland J.(2018) Updates in palliative care-recent advancements in the pharmacological management of symptoms, Clinical Medicine Vol 18, No 1: 11-6

- Temel JS et al. (2010) Early palliative care for patients with metastatic non-small-cell lung cancer. N Engl J Med;363:733-42.

- Temel S et al (2017) Effects of Early Integrated Palliative Care in Patients With Lung and GI Cancer: A Randomized Clinical Trial JCO 35 (8) March 10,2017.

- United Nations. (2014) Strengthening of palliative care as a component of comprehensive care throughout the life course. 67th World Health Assembly. 67/19

- U.S. Census Bureau (2018) Population Projections: Older People Projected to Outnumber Children for First Time in U.S. History. Released No. CB18-41

- WHO (2018). "Palliative Care." from https://www.who.int/news-room/factsheets/detail/palliative-care .

- WHO (2018) Integrating Palliative Care and Symptom Relief into Responses to Humanitarian Emergencies and Crises: A World Health Organization Guide; 2018.

- WHO, (2014) The Worldwide Hospice Palliative Care Alliance. Global atlas of palliative care at the end of life., World Health Organization and The Worldwide Hospice Palliative Care Alliance, Geneva.

- Wood H.; Dickman A.; Star A. and Boland J. (2018) Updates in palliative care - overview and recent advancements in the pharmacological management of cancer pain. Clin Med (Lond). 2018 Feb; 18(1): 17-22. doi: 10.7861/clinmedicine.18-1-17.

- Zimmermann C et al. (2014) Early palliative care for patients with advanced cancer: a cluster randomised controlled trial. Lancet;383:1721-30. 